\title{
Harnessing Diversity in the Face of Environmental Variability: Robustness, Canalization and Genetic Assimilation
}

\section{Luciano Brocchieri}

TB-Seq Inc., San Francisco, CA 94107, USA

*Corresponding author: Brocchieri L, TB-Seq Inc., 953 Indiana Street, San Francisco, CA 94107, USA, Tel: +1 4159263700 ; E-mail: Iuciano@tbseq.com

Received date: July 04, 2017; Accepted date: Aug 08, 2017; Published date: Aug 11, 2017

Copyright: @ 2017 Brocchieri L. This is an open-access article distributed under the terms of the Creative Commons Attribution License, which permits unrestricted use, distribution, and reproduction in any medium, provided the original author and source are credited.

\section{Commentary}

\section{Canalization}

When expression of a phenotype upon a range of perturbations of the genetic background or a variable environmental remains invariant, the phenotypic trait is said to be robust. Robustness of phenotypic characters can be achieved through the evolution of physiological mechanisms that buffer phenotypic variation potentially induced by genetic or environmental variation. The concept of canalization was introduced by Waddington $[1,2]$ to explain a phenomenon by which phenotypic variants are "canalized" into a specific phenotype in a normal range of conditions, specifically in reference to cell fate during the progress of development. An analogy used to describe the processes of canalization and assimilation compares it to formation over time of developmental channels as formed by water falling along a slope, into which different developmental paths are funneled. Severe disturbances may disrupt a channel and cause water to disperse into a myriad of rivulets. Some of these may find the conditions to carve a new channel into which a new set of diverse pathways will then be funneled. The genetic nature of the "channel", as well as the mechanistic aspects of canalization, remain to be determined. Besides its original application to developmental biology, canalization has been invoked in a broader context as a general mechanism to buffer the phenotypic effects of genetic variants, and has been often used as a synonym of robustness [3].

Waddington $[1,2]$ observed that phenotypic variants not expressed under normal conditions can be exposed ("decanalized") as a consequence of environmental stresses. Furthermore, variants originally expressed only under stress, upon artificial or natural selection can be genetically "captured" and expressed also in normal conditions, a phenomenon described as genetic assimilation. The machinery for canalization that provides phenotypic robustness to environmental fluctuations would then also function as an evolutionary capacitor that, in the appropriate conditions, favors evolvability, the ability of an organism to adapt genetically to new environmental conditions. To escape Lamarkism, it must be assumed that the phenotypic variants shielded from the effects of natural selection, and thus allowed to accumulate in the population under canalization, result at least in part from genetic variants rather than uniquely from morphogenic effects of the environment. The process of natural selection would then act on the exposed phenotypic variants resulting first in assimilation of the underlying fittest genetic variants, and then on developing canalization centered on the new phenotypes.

The concept of canalization has been criticized by several authors as Lamarkian in nature, as unlikely to produce adaptive diversification, or as unnecessarily in explaining the process of development or robustness. Williams [4] argued against the likelihood of a role of canalization as a significant player in evolutionary history, suggesting that genetic assimilation is not likely to occur in evolution and, when it does, it is more likely to derive from a condition of adaptive plasticity, the ability of an organism to respond to changing environmental conditions by adaptively modifying its phenotype. Assimilation would develop from plasticity when the environmental conditions that trigger expression of a particular phenotype stabilize for a sufficient period of time and the organism loses the genetic ability to switch to different phenotypes under different environments, thus reverting to a less flexible form of adaptation.

\section{Evolution of rigid canalization in a variable environment}

Rendel [5] proposed a model of canalization by which expression of an optimal phenotype (a "morphogenic substance") depends on the additive effect of a number of genes, the environment, and a regulatory system. The regulatory system is activated when the interaction of the genotype and the environment results in expression of the phenotype above an optimal value $\mathrm{M}_{0}$. The system is physiologically able to buffer the phenotypeup to at most a quantity $\mathrm{m}$ above $\mathrm{M}_{0}$, and thus it is effective in canalizing phenotypes only within the interval $\left[\mathrm{M}_{0}, \mathrm{M}_{0}+\right.$ $\mathrm{m}]$. Rendel's model of rigid canalization assumes a control system that does not react actively to environmental conditions, and in which abnormal phenotypes become exposed only when extreme environmental or genetic conditions expose the physiological limitations of the canalizing system.

Eshel and Matessi [6] studied evolution of a canalization system in a population inhabiting a variable environment. Individuals express a phenotype $P\left(G, E_{m}\right)$ shaped by their genotype $G$ and a "morphogenic environment" $E_{m}$, and their viability is a function of their phenotype and of a selective environment $\mathrm{E}_{\mathrm{s}}$ :

$$
w_{E}(G)=w\left(E_{S}, P\left(G, E_{M}\right)\right)
$$

The selective environment that determines the viability of a phenotype is independent from the environmental factors that, together with the genotype, determine the phenotype. Thus, phenotypic effects caused by environmental morphogenic factors are unrelated to any selective advantage that they may have in the environment in which they are expressed. Eshel and Matessi [6] describe a population of individuals inhabiting a selective, onedimensional environment. A specific environment is described by the value of the optimal phenotype for that environment. Thus, the phenotype $u$ that is optimal in an environment with value $v$ (the " $v$ environment") is $\mathrm{u}=\mathrm{v}$. In other words, the fitness of an individual of phenotype $\mathrm{u}$ in environment $\mathrm{v}$ has optimal value $\mathrm{w}(\mathrm{v}, \mathrm{v})$. Fitness is assumed to depend on the distance $|\mathrm{v}-\mathrm{u}|$ and on a coefficient of selection $1 / \gamma$ according to the Gaussian fitness function: 


$$
w(u, v)=w(v, v) e^{-(u-v)^{2} / 2 \gamma^{2}}
$$

Environments are assumed to follow a standardized normal distribution with mean $\mathrm{v}=0$ and variance $\sigma^{2}=1$ and phenotypes are also normally distributed around their average with some variance $\sigma^{2}$. Eshel and Matessi find that the mean viability $w(u)$ of a phenotype $u$ randomly exposed to the variable environment is maximized when $\mathrm{u}=$ 0 [6]. Thus, positive selection is expected to drive evolution of $\mathrm{u}$ toward its optimal value $u=0$ and to purify genetic variants to mutationselection equilibrium, minimizing phenotypic variance. However, if phenotypic variance is determined independently by genetic and environmental factors it can be expressed as:

$$
\sigma^{2}=\sigma^{2}\left(P\left(G, E_{M}\right)\right)=\sigma_{G}^{2}+\sigma_{E}^{2}
$$

i.e., the sum of a $\sigma_{G}^{2}$ component due to genetic factors, and a $\sigma_{E}^{2}$ component due to the morphogenic environment EM. Purifying selection will only be able to act on the genetic component $\sigma_{G}^{2}$ of the phenotypic variance, and residual phenotypic variance due to the morphogenic-environment component $\sigma_{E}^{2}$ will always be present above mutation-selection balance. This residual variance can provide selective pressure for the evolution of a canalization system [6]. The canalization system will promote accumulation of cryptic phenotypic variability generated by the morphogenic environment, shielding it from natural selection. If the same system has also the side effect of suppressing phenotypic variability generated by genotypic variants (the "congruence" hypothesis [7]), it will also promote accumulation of genetic variability.

\section{Evolution of adaptive canalization in a variable environment}

Different types of phenotypic modifiers can be categorized depending on their ability to respond to the state of the environment and of the primary phenotype. The system of rigid canalization described above is a rudimentary modifier that cannot respond to environmental signals and will be most effective by mapping any phenotype deviation of the trait to its optimal value $[5,6]$. A much more effective system would activate or deactivate canalization of a phenotype $u$ in relation to the value of the environment, i.e., expressing the decanalized phenotype every time the environment $\mathrm{v}$ is distant from its average value $\mathrm{v}=0$ and the phenotype $\mathrm{u}$ is comparatively close to $\mathrm{v}$. This sophisticated mechanism of regulation would likely require a long process to evolution, leading to genetic plasticity.

Building on the model described above, Eshel and Matessi [6] envision evolution of a system of adaptive canalization, which has the ability of activating or deactivating canalization of the phenotype in response to environmental conditions. Adaptive canalization only responds to environmental stimuli irrespective of the value of the phenotype, thus excluding the case of a system that regulates canalization based on information from both the environment and the primary phenotype, i.e., that can expose or hide a primary phenotype in relation to its adaptive value to specific environments. The latter would correspond to adaptive plasticity, a more sophisticated form of adaptation than adaptive canalization (see [8] for a review). Eshel and Matessi [6] study the conditions under which the evolution of an adaptive canalization system in a variable environment is favored by natural selection and addressing the objections by Williams [4] that the phenotypic variants exposed by a stressful environment are unlikely to provide adaptations to a new environment. The canalization policy (i.e., the decision of implementing or not canalization in relation to the environment) is considered to be a phenotype controlled by genes, and thus also subject to natural selection under the selective environment $\mathrm{E}_{s}$.

It is reasonable to imagine evolution of a system of adaptive canalization that responds to environmental stimuli by inactivating canalization whenever the selective environment is sufficiently different from its expected value $\mathrm{Ev}=0$, i.e., when the canalized phenotype $(\mathrm{u}=0)$ is far from the optimal phenotype $(\mathrm{u}=\mathrm{v})$ for that environment. However, Williams suggested [4] that inactivation of canalization in extreme environments can easily cause more disadaptive phenotypes than random adaptations to the different environment. In fact, Eshel and Matessi also point out that the expected square distance $E(v-u)^{2}$ of environment $\mathrm{v}$ from a random phenotype $\mathrm{u}$, is always greater than the expected square distance $E v^{2}$ of the environment from its expectation. The average fitness of a random individual drawn from a primary phenotype with mean $u$ and variance $\sigma^{2}$ when exposed to an environment $\mathrm{v}$ can be precisely calculated and compared to the fitness of the canalized phenotype. Assume a canalization system that maps a trait-value $u$ to a modified value $\theta(u)$, such that for all $\mathrm{u}$ full canalization corresponds to $\theta(u)=0$, and complete deactivation of canalization corresponds to $\theta(u)=u$. The average fitness in environment $\mathrm{v}$ of the phenotype $\theta(u)$ can be obtained in the general case integrating fitness over all possible values $u$ of the primary phenotype: $w_{\theta(u)}(v)=\frac{w(v, v)}{\sqrt{2 \pi \sigma^{2}}} \int_{-\infty}^{+\infty} e^{-\frac{[\theta(u)-v]^{2}}{2 \gamma^{2}}} e^{-\frac{u^{2}}{2 \sigma^{2}}} d u$

Using this general expression, the mean fitness in environment $\mathrm{v}$ of fully canalized individuals can be calculated imposing $\theta(u)=0$, as: $w_{0}(v)=w(v, v) e^{-\frac{v^{2}}{2 \gamma^{2}}}$

Substituting $\theta(u)=u$ instead gives the average fitness of individuals when canalization is deactivated: $w_{u}(v)=\frac{\gamma w(v, v)}{\sqrt{\sigma^{2}+\gamma^{2}}} e^{\frac{-v^{2}}{2\left(\sigma^{2}+\gamma^{2}\right)}}$

Deactivation of canalization would be then favored in the selective environment $\mathrm{v}$ when $w_{u}(v)>w_{0}(v)$. Substituting the two previous expressions and rearranging, this condition is verified when [6]: $v^{2}>\frac{\gamma^{2}\left(\sigma^{2}+\gamma^{2}\right)}{\sigma^{2}} \ln \left(1+\frac{\sigma^{2}}{\gamma^{2}}\right)=\hat{v}^{2}$.

Thus, inactivation of canalization is indeed favored provided that the environment is more distant from its optimal value than a threshold value. This result shows that an adaptive canalization system that responds to environmental conditions in the absence of information on the adaptive value of the primary phenotype can evolve and is preferable to a system of rigid canalization. In particular, it implies that when random phenotypes are exposed to severe environmental stresses, the advantage of exposing certain deviations in the appropriate direction from the mean phenotype overcomes the selective disadvantage of exposing deviations of the primary phenotype in the opposite direction from the mean. In conclusion, 
when phenotypic variants are randomly distributed in a variable selective environment, selection will favor evolution of an adaptive canalization system that can not only neutrally protects genetic variants of the primary phenotype, but will also be able to expose genetic variants that can be positively selected. This will provide the conditions for the primary phenotype and the canalization system to coevolve under the pressure of natural selection.

\section{Coevolution of adaptive canalization and primary phenotype}

Eshel and Matessi [6] study the coevolution of primary phenotype and canalization considering the viability of genotypes that control: (i) the mean primary phenotype $y$, and (ii) a canalization policy $\lambda$. The value $u$ of the primary phenotype of an individual of genotype $(\lambda, y)$ is drawn from a normal distribution with mean y and variance $\sigma^{2}$. The canalization policy $\lambda(\mathrm{v})$ determines the decision, based on environment $\mathrm{v}$, of either implementing full canalization of the phenotype or of inactivating canalization. With this setup, Eshel and Matessi identify among genotypes $(\lambda, y)$ the "unbeatable strategy" that maximizes the fitness $\mathrm{W}(\lambda, \mathrm{y})$ when exposed to a random environment. Given environment $v$, the viability of the canalized phenotype is:

$$
S_{0}=(y, v)=e^{-v^{2} / 2 \gamma^{2}}
$$

When canalization is inactivated, individuals will show a distribution of phenotypes around $\mathrm{y}$, and in environment $\mathrm{v}$ their average viability will be:

$$
s_{1}(y, v)=\frac{\gamma}{\sqrt{\sigma^{2}+\gamma^{2}}} e^{-(v-y)^{2} / 2\left(\sigma^{2}+\gamma^{2}\right)} .
$$

In environment $\mathrm{v}$ it is then best to canalize the expected primary phenotype y whenever or, explicitly, when:

$$
\ln \left(\frac{\gamma^{2}+\sigma^{2}}{\gamma^{2}}\right) \geq \frac{v^{2}}{\gamma^{2}}-\frac{(v-y)^{2}}{\gamma^{2}+\sigma^{2}}
$$

This condition defines an interval $\left[v_{-}, v_{+}\right]$of environments within which a canalized phenotype has higher fitness than a non-canalized phenotype (Fig 1), and a corresponding optimal canalization policy $\hat{\lambda}_{y}$ given the primary phenotype $y$. The interval of canalization widens with increasing variance of the phenotype distribution, is symmetric around $\mathrm{v}=0$ when $\mathrm{y}=0$, and extends further to the side opposite to the sign of $y$ when $y \neq 0$ (Fig. 1). Eshel and Matessi find that, if the variance of the primary phenotype is large enough, the unbeatable strategy that maximizes $W\left(\hat{\lambda}_{y}, y\right)$ over y always corresponds to the mean phenotype $y^{0}=0$. However, if the variance of the primary phenotype is small then the mean phenotype will tend to shift toward one of two finite values $\pm y^{0}$, with $y^{0}>0$. Thus, natural selection will favor the evolution of a system of adaptive canalization, and of shifts in the primary phenotype in sufficiently unstable environments.

The fundamental result of the analysis of Eshel and Matessi is that even when there is no mechanism in place to adaptively express phenotypic variants in relation to changes in the environment, an adaptive mechanism of canalization can evolve in response to environmental stimuli. Contrary to Williams' prediction, this result suggests that canalization will favor the development of phenotypes that can be enriched when exposed by de-canalization to rare environmental niches. These in turn can provide preadaptations to catastrophic events resembling environmental conditions previously experienced in some environmental niche, leading to a process of punctuated evolution [6]. A new canalization system should be then expected slowly to evolve, allowing hidden variability to be generated around the new optimal phenotype.

\section{Canalization, intrinsic robustness, and evolvability}

The work of Eshel and Matessi [6] provides theoretical support to the idea that a mechanism of adaptive canalization that responds to environmental conditions irrespective of the state of the primary phenotype provides increased viability to a population inhabiting a variable environment. Furthermore, it shows that adaptive canalization can result in enrichment in genetic variants associated with phenotypes that have been exposed to local environmental fluctuations and these may provide significant pre-adaptations upon major shifts of the whole environment towards any of the previously encountered conditions, facilitating a process of punctuated evolution $[9,10]$. Key to the role of canalization as an evolutionary capacitor is the assumption that at least part of the canalized phenotypic variants are generated by genetic variants that are selected upon decanalization [6]. However, as we have seen a canalization system is more likely to evolve when phenotypic variability is generated by the morphogenic environment. Evolution of canalization is favored by strong selection against the phenotypic expression of genetic variants that, however, are rapidly eliminated by stabilizing selection. Thus, canalization could evolve as an evolutionary capacitor only if strong selection against genetic variants is balanced by a very high mutation rate [11] or if a canalization system that evolves under the selective pressure of morphogenic variants can extend its buffering activity also to genetic variants [6].

Alternative hypotheses to Waddington's canalization explanation for assimilation have gained considerable interest in more recent years. In this respect, it is worthwhile to make clear a distinction between "canalization" and other forms of phenotypic robustness. Hermisson and Wagner [12] propose to restrict the definition of canalization to adaptive robustness, i.e., the buffering of a trait resulting from a process of natural selection. In contrast, intrinsic robustness arises as a by-product of selection for some other property of the primary trait. A leading hypothesis is that phenotypic buffering and assimilation can be achieved without resorting to canalization if the primary trait is under the control of multiple genes of a quantitative-trait locus (QTL) $[13,14]$. In normal environmental conditions all genetic variants resulting by different combination of alleles (genotypes) would produce the same wild-type phenotype. Stressful envronmental conditions however would lower a threshold above which an alternative phenotype become expressed. The alleles most sensitive to the new environmental conditions can then be selected and once environmental conditions become normal the increased frequency of the selected alleles will be sufficient to still observe the new phenotype (assimilation). The assumption that a unique wild-type phenotype is expressed in normal conditions by different genotypes, describes an instance of phenotypic robustness over genetic perturbations emerging as an intrinsic property of the system rather than from selection for a separate canalization system.

Robustness of phenotypes to environmental and genetic stressors has been described also as an emergent property of complex genetic networks associated with developmentally stable phenotypes [15-17]. It has been shown that selection for stable genetic networks not only 
results in a phenotype robust to mutations [16], but is also sufficient for producing genetic assimilation of alternative phenotypes observed after strong perturbations, irrespective of the presence of selection for a specific stable phenotype [17].

The role of mechanisms of robustness as evolutionary capacitors [18] is very much debated [19-22]. In particular, while examples of robustness to environmental perturbations abound [3,19], their relevance in buffering genetic perturbations still attracts much debate $[19,23]$. Frazer and Schadt [24] used genetic mapping approaches to identify QTL polymorphisms associated with environmental robustness (ER) or to genetic robustness (GR), i.e., loci whose genetic perturbation leads to changes in primary-trait variance over environmental or genetic perturbations. Association studies have been criticized for methodological and conceptual limitations (see [25] for an interesting perspective), and can be misleading in interpreting effects on robustness (see [19] for a critical review focusing on the role of epistatic effects). However, it is interesting that Frazer and Schadt [24] identified many polymorphisms associated with changes in GR or ER. A relevant conclusion was that loci associated with ER are generally independent from those controlling GR, suggesting that mechanisms of genetic robustness evolve independently from those involved in environmental robustness. Another finding was that GR systems largely act in cis, whereas ER systems act predominantly in trans. Although no distinction was made between intrinsic and adaptive robustness, this result might suggest that changes in GR could identify cis-acting intrinsic robustness, whereas changes in ER might more likely represent systems of trans-acting adaptive robustness (canalization). The identification and interpretation of possible mechanisms that enable robustness of different biological systems appears to grow as diverse as the number of systems that have been investigated [3], and the debate on how robustness against environmental and genetic variability is achieved and contributes to evolvability continues to these days [19].

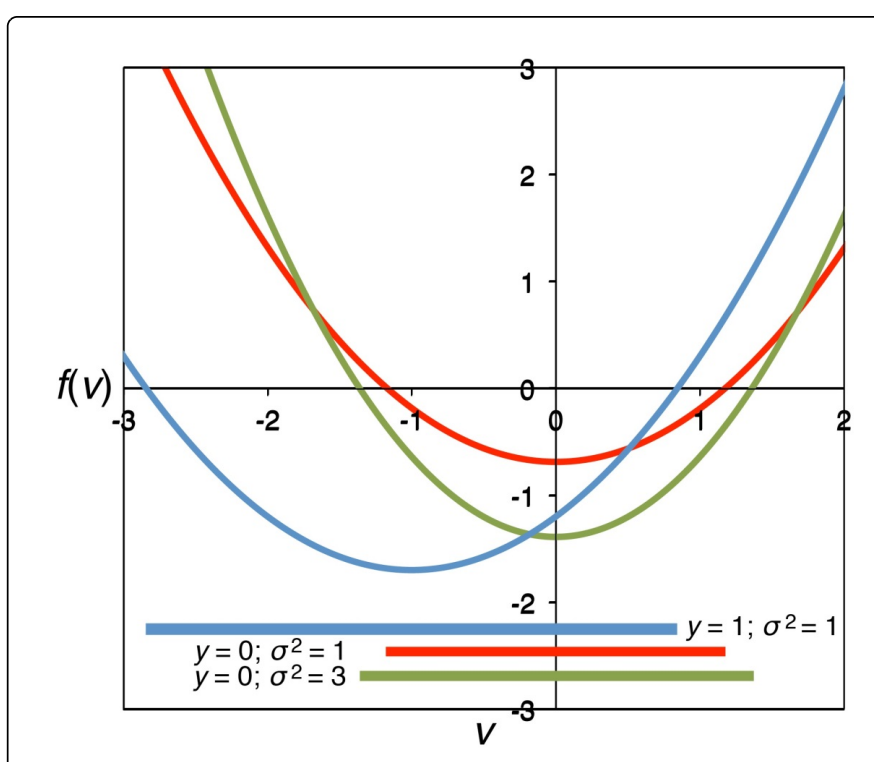

Figure 1: Environmental regions in which a trait canalized to its optimal value $y=0$ has higher fitness than a trait distribution of mean $y$ and variance $\sigma^{2}[6]$. Coefficient of selection is set to 1.0. The function $f(v)$ represents the difference in fitness between a decanalized and a canalized phenotype in different environments $\mathrm{v}$. Intervals of environments favoring canalization become wider with increasing phenotypic variance. When the expected phenotype y is different from 0 (i.e., different from the optimal environment $v=0$ ) the interval of canalization extends more on the opposite side of the sign of $y$ (see main text).

\section{References}

1. Waddington $\mathrm{CH}$ (1952) Selection of the genetic basis for an acquired character. Nature 169: 625-626.

2. Waddington $\mathrm{CH}$ (1953) Genetic assimilation of an acquired character. Evolution 7: 118-126.

3. Félix MA, Barkoulas M (2015) Pervasive robustness in biological systems. Nature Rev: Genetics 16: 483-496.

4. Williams GC (1966) Adaptation and Natural Selection. Princeton University Press, Princeton, NJ, USA pp: 1-307.

5. Rendel JM (1967) Canalisation and gene control. Logos Press, London.

6. Eshel I, Matessi C (1998) Canalization, genetic assimilation and preadaptation: a quantitative genetic model. Genetics 149: 2119-2133.

7. Meiklejohn CD, Hartl DL (2002) A single mode of canalization. Trends Ecol Evol 17: 468-473.

8. Ghalambor CK, McKay JK, Carroll SP, Reznick DN (2007) Adaptive versus non-adaptive phenotypic plasticity and the potential for contemporary adaptation in new environments. Funct Ecol 21: 394-407.

9. Eldredge N (1971) The allopatric model and phylogeny in Palaeozoic invertebrates. Evolution 25: 156-167.

10. Eldredge N, Gould SJ (1972) Punctuated equilibria: an alternative to phyletic gradualism. In: Schopf TJM (ed.) Models in Paleobiology. Freeman and Cooper, San Francisco pp: 82-115.

11. Wagner GP, Booth G, Bagheri-Chaichian H (1997) A population genetic theory of canalization. Evolution 51: 329-347.

12. Hermisson J, Wagner GP (2005) Evolution of phenotypic robustness. In: Robust Design: A Repertoire from Biology, Ecology, and Engineering, Jen E (ed.), Oxford University Press, Oxford, pp 47-70. 
Citation: Brocchieri L (2017) Harnessing Diversity in the Face of Environmental Variability: Robustness, Canalization and Genetic Assimilation. J Phylogenetics Evol Biol 5: 182. doi:10.4172/2329-9002.1000182

Page 5 of 5

13. Scharloo W (1991) Canalization - genetic and developmental aspects. Annu Rev Ecol Syst 22: 65-93.

14. Falconer DS, Mackay TFC (1996) Introduction to quantitative genetics. Longman, Essex, UK pp: 309-310.

15. Wagner A (1996) Does evolutionary plasticity evolve. Evolution (Lawrence, Kans) 50: 1008-1023.

16. Siegal ML, Bergman A (2002) Waddington's canalization revisited: developmental stability and evolution. Proc Natl Acad Sci USA 99: 10528-10532.

17. Masel J (2004) Genetic assimilation can occur in the absence of selection for the assimilating phenotype, suggesting a role for the canalization heuristic. J Evol Biol 17: 1106-1110.
18. Rutherford SL, Lindquist S(1998) Hsp90 as a capacitor for morphological evolution. Nature 396: 336-342.

19. Siegal ML, Leu JY (2014) On the nature and evolutionary impact of phenotypic robustness mechanisms. Ann Rev Ecol Evol Syst 45: 496-517.

20. Masel J (2013) Q\&A: evolutionary capacitance. BMC Biol 11: 1-4

21. Hermisson J, Wagner GP (2004) The population genetic theory of hidden variation and genetic robustness. Genetics 168: 2271-2284.

22. Siegal ML (2013) Crouching variation revealed. Mol Ecol 22: 1187-1189.

23. Masel J, Siegal ML (2009) Robustness: mechanisms and consequences. Trends Genet 25: 395-403.

24. Fraser HB, Schadt EE (2010) The Quantitative Genetics of Phenotypic Robustness. PLoS ONE 5: e8635.

25. Boyle EA, Li YI, Pritchard JK (2017) An Expanded View of Complex Traits: From Polygenic to Omnigenic. Cell 169: 1177-1186. 